\title{
Antibiotic "Timeout" on Electronic Prescribing - Impact of Antibiotic Hardstop on Antimicrobial Stewardship Initiatives
}

Semun Galimam ( $\square$ Semun.galimam@health.nsw.gov.au )

Central Coast Local Health District

Brydon Panozzo

Central Coast Local Health District

Kieran Muir

Central Coast Local Health District

Ruchir Chavada

Pathology North Central Coast

\section{Research Article}

Keywords: antibiotic, timeout, antimicrobial, stewardship, electronic

Posted Date: August 24th, 2021

DOI: https://doi.org/10.21203/rs.3.rs-806552/v1

License: (c) (i) This work is licensed under a Creative Commons Attribution 4.0 International License. Read Full License 


\section{Abstract}

Background: Antimicrobial resistance (AMR) remains a major public health threat and the exploration of interventions which may reduce inappropriate antimicrobial use are of particular interest. An Antibiotic Timeout (ATO) was included within the electronic medicine (eMeds) system introduced to the Central Coast Local Health District (CCLHD) in 2018. The function allows prescribers to set a predetermined time at which antibiotic orders would cease. By default, the function set prescribed length to 5 days with a view to encourage prescribers to review existing antimicrobial orders and reduce inappropriate use.

Methods: Records of adult inpatients prescribed broad spectrum antimicrobials with a registered indication of community acquired pneumonia (CAP) or an infective exacerbation of chronic obstructive pulmonary disease (IECOPD) between the $1^{\text {st }}$ of March 2017 and 31st May 2017 for the pre eMeds cohort and $1^{\text {st }}$ March 2019 and 31st May 2019 for the post eMeds cohort were randomly selected from our local health network's Guidance MS ${ }^{\circledR}$ system. Baseline demographics, antimicrobial prescribing records and documented adverse events related to the antibiotic timeout function were collated/analysed. The days of therapy (DOT) and length of therapy (LOT) for each encounter were calculated manually and results analysed using a two-tailed t-test or Mann-Whitney $\mathrm{U}$ test.

Results: Of patients eligible to have the ATO function activated during their admission, $34 \%(n=34)$ had the function deployed at least once. Following the introduction of eMeds mean DOT for the pooled indications cohort was reduced by 3.02 days $(\mathrm{Cl} 95 \% 0.41-5.63, p<0.05)$ and mean LOT by 1.97 days (Cl $95 \% 0.39-3.55, p<0.05)$. The timeout function resulted in 2 cases of delayed or unintentionally ceased therapies.

Conclusions: Following the introduction of electronic prescribing and ATO, a significant reduction was observed in the DOT and LOT for antimicrobial use for inpatients with CAP and IECOPD without a significant increase in adverse events. Further research is required to determine the extent to which the antibiotic timeout functionality directly contributed to this effect and if the effect is present across a broader range of indications.

\section{Key Points/summary}

- Local health district in regional NSW

- Electronic prescribing introduced with Antibiotic Timeout function

- Defaulted to a 5-day therapy course

- Significant reduction in antimicrobial use observed for CAP and COPD

- No significant increase in adverse events.

\section{Introduction}


Antimicrobial Resistance (AMR) poses an increasing threat to public health that necessitates urgent action. Around the globe a broad variety of strategies are being implemented in order to combat the issue [1]. Antimicrobial Stewardship (AMS) describes a system of activities and methodologies which aim to optimise antimicrobial use and maximise clinical outcomes while minimising unintended consequences, including the emergence of AMR [2]. A number of Information Technology (IT) tools have been utilised to facilitate AMS including computerised decision support systems, antimicrobial approval systems and surveillance systems [3]. The establishment of eMeds prescribing has opened up new opportunities to facilitate AMS [4]. The use of eMeds with integrated AMS functionality has been associated with a variety of positive outcomes including increased adherence to prescribing guidelines and a reduction in antimicrobial prescribing overall. Software functions which have been integrated into eMeds systems to facilitate AMS have included antimicrobial timeouts, prescribing alerts and indication order sets $[5,6]$.

Whilst there is sparse evidence to provide guidance on optimal timeout processes in general, the literature describing which individual features regarding timeout functions specific to eMeds and which are likely to have the greatest impact on reducing rates of inappropriate antimicrobial prescribing is inconsistent. Internationally, hospitals using an Antibiotic Timeout (ATO) automated alert in eMeds to identify patients that have received over 48 hours of antimicrobial therapy have shown significantly decreased DOT of broad-spectrum antimicrobials $[7,8]$. However, there is conflicting evidence within studies looking into the efficacy of EMR ATO automated alerts in specific patient populations [9]. Furthermore, the large majority of the literature describing ATO functions have been set in the US, Europe and northern Asia with negligible studies describing ATO interventions in an Australian context [10].

Community Acquired Pneumonia (CAP) and Infective Exacerbation of Chronic Obstructive Pulmonary Disease (IECOPD) both represent infective illnesses with a high disease burden and are indications for which broad-spectrum antimicrobials are commonly prescribed $[11,12]$. Short course antibiotic therapy has demonstrated non-inferiority to longer courses of therapy for both indications and there is ongoing interest in understanding the impacts of short course antimicrobial therapy on clinical outcomes and antimicrobial resistance rates $[13,14]$. The annual National Antimicrobial Prescribing Survey (NAPS) conducted in the CCLHD has consistently shown inappropriate antimicrobial use in the treatment of CAP and IECOPD due to prolonged duration of therapy. For these reasons, CAP and IECOPD were deemed suitable indications upon which to evaluate the effect of antibiotic timeout on antimicrobial prescribing behaviour.

\section{Methods}

The Central Coast of NSW has 2 hospitals with 873 beds and serves population of approximately 350,000. The CCLHD introduced the antibiotic timeout function into eMeds in February/March 2018. The antibiotic timeout function allows prescribers to select a predetermined time at which the chosen medication order will cease unless the prescriber intervenes, or a new order is placed. Antimicrobials at CCLHD are grouped into a traffic light system with red encompassing antimicrobials which requiring consultation with and approval by an Infectious Diseases physician or clinical microbiologist as well as 
registration on our electronic decision support and approval system Guidance MS® prior to prescribing. Orange antimicrobials require registration in our Guidance $\mathrm{MS}{ }^{\circledR}$ system and green antimicrobials are not routinely monitored. Red and orange antimicrobials represent either broad spectrum or high-risk antimicrobials. By default, antibiotic timeouts are set to 5 days, with prescribers having the option to disable this feature at the time of prescribing or during the course of the order.

This study was designed as a pre-post interventional study. One hundred records prior to the introduction of eMeds from the period 01/03/2017-31/05/2017 of adult inpatients prescribed broad spectrum antimicrobials with a registered indication of CAP or IECOPD were randomly selected from our Guidance MS ${ }^{\circledR}$ system. The random selection process was repeated for the period 01/03/2019-31/5/2019 which followed the introduction of eMeds to CCLHD. Baseline demographics, antimicrobial prescribing data and any documented adverse events related to the antibiotic timeout function were collated and analysed. Statistical analysis was performed using Stata Statistical Software: Release 16 (College Station, TX: StataCorp LP, 2019) with Days of Therapy (DOT) and Length of Therapy (LOT) calculated according to Public Health Ontario Antimicrobial Stewardship guidance [15].

Non-normal distribution was confirmed using Shapiro-Wilk tests for LOT and DOT subgroups $(p=0.00$ for both measures), for pre eMeds and post eMeds subgroups ( $p=0.00$ for both measures) and CAP and IECOPD subgroups ( $p=0.00$ for both measures). The data naturally follows a non-normal distribution as the likelihood of a patient being on an antimicrobial is generally inversely related to the number of days a patient has been on the antimicrobial. LOT and DOT median and interquartile ranges were calculated using a two-sample t-test on the raw data. Due to the non-normal distribution of data and smaller sample sizes on subgroup analysis a two sample Mann-Whitney $U$ test was used to confirm statistical significance.

\section{Results}

The pre eMeds and post eMeds cohort were of comparable ages, comorbidities, rates of bacteraemia, and rates of involvement of an infectious diseases physician (Table 1). There was a small but statistically significant difference in the number of patients requiring intensive care unit (ICU) admission between the pre eMeds and post eMeds cohorts (20 vs 10 patients, $p=0.047$ ) and patients from Residential Aged Care Facilities (RACF) in the (18 vs 30 patients, $p=0.047$ ). A CURB-65 analysis of respiratory infection severity showed no significant difference between the pre eMeds group mean (SD) of 2.98 (1.21) and post eMeds group mean (SD) of 3.02 (1.18) (mean difference - 0.04 severity rating, [95\% $\mathrm{Cl},-0.374-0.294$ ], $\mathrm{p}=0.814$ ) (Table 4). Analysis showed no significant difference in infection severity between the CAP group mean (SD) of 2.98 (1.25) and IECOPD group mean (SD) of 2.06 (1.03) (mean difference - 0.077 severity rating, [95\% $\mathrm{Cl},-0.452-0.301], p=0.689)$. 
Table 1

Baseline characteristics of Pre eMeds and Post eMeds cohorts

\begin{tabular}{|c|c|c|c|}
\hline & Pre eMeds & Post eMeds & p-value \\
\hline Total Patients & 100 & 100 & \\
\hline Age in years, median (IQR) & $74.5(62-84)$ & $77(67-87)$ & 0.1718 \\
\hline RACF & 18 & 30 & $0.0469 *$ \\
\hline Bacteraemia & 3 & 5 & 0.4705 \\
\hline CKD & 10 & 19 & 0.0707 \\
\hline T2DM & 17 & 28 & 0.0625 \\
\hline Respiratory Disease & 55 & 53 & 0.7766 \\
\hline PVD & 2 & 5 & 0.2484 \\
\hline Immunosuppressed & 7 & 13 & 0.1573 \\
\hline Haematological Malignancy & 5 & 5 & 1.0000 \\
\hline ICU Admission & 20 & 10 & $0.0477^{*}$ \\
\hline Directed Therapy & 19 & 12 & 0.1714 \\
\hline ID Involvement & 21 & 14 & 0.1927 \\
\hline CURB65 mean (SD) & $2.98(1.21)$ & $3.02(1.18)$ & 0.814 \\
\hline \multicolumn{4}{|l|}{ CURB65 Categorised (\%) } \\
\hline $0-1$ & 12 & 10 & 0.6513 \\
\hline 2 & 23 & 19 & 0.4874 \\
\hline$\geq 3$ & 65 & 71 & 0.3631 \\
\hline \multicolumn{4}{|c|}{$\begin{array}{l}\text { P-value calculated using two-sample test of proportions for categorical variables and two-sample t- } \\
\text { test for continuous variables }\end{array}$} \\
\hline \multicolumn{4}{|c|}{$\begin{array}{l}\text { Abbreviations: SD, standard deviation; CKD, chronic kidney disease; T2DM, type } 2 \text { diabetes mellitus; } \\
\text { PVD, peripheral vascular disease; ICU, intensive care unit; ID, infectious diseases, RACF, Residential } \\
\text { Aged Care Facility }\end{array}$} \\
\hline
\end{tabular}

Multiple classes of antimicrobials were used in both patient cohorts. There was statistically significant lower rate of benzylpenicillin ( 0.074 vs $0.027,[95 \% \mathrm{Cl}, 0.014-0.081] \mathrm{p}=0.0076)$, ciprofloxacin $(0.034 \mathrm{vs}$ $0.010,[95 \% \mathrm{Cl}, 0.002-0.046], \mathrm{p}=0.043)$ and piperacillin-tazobactam $(0.092$ vs $0.272,[95 \% \mathrm{Cl}, 0.029-$ 
$0.100], p=0.001)$ use in the post eMeds cohort (Table 2$)$. There was additionally a higher rate of ceftriaxone prescription in the post eMeds cohort (0.21 vs 0.29 , [95\% Cl, $-0.144--0.010], p=0.024)$. 
Table 2

Antibiotic use

\begin{tabular}{|c|c|c|c|}
\hline \multirow[t]{2}{*}{ Antimicrobial } & \multicolumn{3}{|c|}{$\begin{array}{l}\text { Number of patients prescribed antimicrobial during } \\
\text { admission }\end{array}$} \\
\hline & Pre eMeds & Post eMeds & \\
\hline Amoxicillin-Clavulanate & $31(8 \%)$ & $15(5 \%)$ & $p=0.064$ \\
\hline Amoxicillin & $7(2 \%)$ & $8(3 \%)$ & $p=0.64$ \\
\hline Ampicillin & $4(1 \%)$ & $1(1 \%)$ & $p=0.25$ \\
\hline Azithromycin & $30(9 \%)$ & $38(13 \%)$ & $p=0.075$ \\
\hline Benzylpenicillin & $26(7 \%)$ & $8(3 \%)$ & $\begin{array}{l}p= \\
0.0076^{*}\end{array}$ \\
\hline Cefalexin & $5(1 \%)$ & $1(1 \%)$ & $p=0.15$ \\
\hline Cefazolin & $4(1 \%)$ & $0(0 \%)$ & $p=0.066$ \\
\hline Ceftazidime & $0(0 \%)$ & $2(1 \%)$ & $p=0.12$ \\
\hline Ceftriaxone & $74(21 \%)$ & $85(29 \%)$ & $p=0.024 *$ \\
\hline Cefuroxime & $12(3 \%)$ & $19(6 \%)$ & $p=0.075$ \\
\hline Ciprofloxacin & $12(3 \%)$ & $3(1 \%)$ & $p=0.043^{*}$ \\
\hline Clindamycin & $2(1 \%)$ & $1(1 \%)$ & $p=0.67$ \\
\hline Doxycycline & $56(16 \%)$ & $56(19 \%)$ & $p=0.32$ \\
\hline Flucloxacillin & $7(2 \%)$ & $10(3 \%)$ & $p=0.27$ \\
\hline Gentamicin & $15(4 \%)$ & $22(7 \%)$ & $p=0.084$ \\
\hline Meropenem & $3(1 \%)$ & $1(1 \%)$ & $p=0.40$ \\
\hline Metronidazole & $12(3 \%)$ & $7(2 \%)$ & $p=0.43$ \\
\hline Moxifloxacin & $2(1 \%)$ & $3(1 \%)$ & $p=0.52$ \\
\hline Piperacillin-Tazobactam & $32(9 \%)$ & $8(3 \%)$ & $\begin{array}{l}p= \\
0.0007 *\end{array}$ \\
\hline Tobramycin & $1(1 \%)$ & $1(1 \%)$ & $p=0.90$ \\
\hline $\begin{array}{l}\text { Trimethoprim- } \\
\text { Sulfamethoxazole }\end{array}$ & $7(2 \%)$ & $2(1 \%)$ & $p=0.15$ \\
\hline
\end{tabular}

P-value calculated using two-sample proportions test (z-test) *statistically significant $p$-value $(p \leq 0.05)$ 


\begin{tabular}{|c|c|c|c|}
\hline \multirow[t]{2}{*}{ Antimicrobial } & \multicolumn{3}{|c|}{$\begin{array}{l}\text { Number of patients prescribed antimicrobial during } \\
\text { admission }\end{array}$} \\
\hline & Pre eMeds & Post eMeds & \\
\hline Trimethoprim & $0(0 \%)$ & $1(1 \%)$ & $p=0.28$ \\
\hline Vancomycin & $7(2 \%)$ & $2(1 \%)$ & $p=0.15$ \\
\hline Total & $349(100 \%)$ & $294(100 \%)$ & \\
\hline \multicolumn{4}{|c|}{ P-value calculated using two-sample proportions test (z-test) } \\
\hline *statistically s & $(p \leq 0.05)$ & & \\
\hline
\end{tabular}

Following the introduction of electronic prescribing antimicrobial mean DOT was reduced by a mean 3.07 days $(\mathrm{Cl} 95 \%, 0.47-5.67, \mathrm{p}<0.05)$. The mean LOT was reduced by 2.00 days $(\mathrm{Cl} 95 \%, 0.42-3.57, \mathrm{p}<$ 0.05). On subgroup analysis the reduction in DOT and LOT for the indication of CAP was not maintained, $p=0.093$ and $p=0.06$ respectively (Table 3 ). Additionally, no difference in the DOT or LOT for the indication of IECOP was identified, $p=0.225$ and $p=0.165$ respectively. It is likely that this is secondary to underpowering upon stratification for subgroup analysis.

Table 3

Subgroup Analysis Pre vs Post eMeds

\begin{tabular}{|c|c|c|c|}
\hline & \multicolumn{3}{|l|}{ CAP } \\
\hline & Pre eMEDS & Post eMEDS & \\
\hline Number of Cases & 65 & 82 & \\
\hline DOT (days) - MEDIAN (IQR) & $12(8-17)$ & $10(7-15)$ & $p=0.093$ \\
\hline LOT (days) - MEDIAN (IQR) & $7(4-12)$ & $6(4-9)$ & $p=0.06$ \\
\hline \multicolumn{4}{|l|}{ IECOPD } \\
\hline & Pre eMEDS & Post eMEDS & \\
\hline Number of Cases & 35 & 18 & \\
\hline DOT (days) - MEDIAN (IQR) & $12(7-18)$ & $10.5(8-12)$ & $p=0.225$ \\
\hline LOT (days) - MEDIAN (IQR) & $7(4-9)$ & $5(4-7)$ & $p=0.165$ \\
\hline
\end{tabular}

34 of 100 post eMeds patients had the antibiotic timeout function activated at least once during their admission (Table 5). Of those 34 patients, 10 had their antimicrobial continued by the team, 12 patients had their antimicrobial intentionally discontinued by the team (as documented in patient notes), 10 patients had their IV antimicrobials changed to PO and 2 patients had either a dose of antimicrobial missed/delayed, or their regimen unintentionally ceased. Of these 2 patients with missed/delayed doses or unintentionally ceased antimicrobial regimens, 1 patient missed 24 hours' worth of antimicrobials and was then changed to PO and 1 patient missed 48 hours' worth of antimicrobials and was then restarted 
on the same regimen with nil documented adverse outcomes. The outcomes and contributing factors of these two instances of patients having unintentional interruptions/discontinuations of therapy were reported to the CCLHD AMS Subcommittee and clinical governance units with subsequent remedial actions taken including ongoing medical officer education and clinical team awareness.

Table 4

CURB65 score pre eMeds vs post eMeds

\begin{tabular}{|llll|}
\hline CURB65 score & Pre eMeds & Post eMeds & Total \\
\hline 0 & 1 & 2 & 3 \\
\hline 1 & 11 & 8 & 19 \\
\hline 2 & 23 & 19 & 42 \\
\hline 3 & 31 & 41 & 72 \\
\hline 4 & 22 & 17 & 39 \\
\hline 5 & 12 & 13 & 25 \\
\hline Total & 100 & 100 & 200 \\
\hline Mean (SD) & $2.98(1.21)$ & $3.02(1.18)$ & $3(1.19)$ \\
\hline
\end{tabular}

Table 5

Antibiotic Timeout Activation Outcomes

\section{Antibiotic Timeout Action}

Patient encounters in which the antibiotic timeout is activated at least once

Antimicrobial continued by the team

Antimicrobial intentionally discontinued by the team

Antimicrobials changed from intravenous to per oral route

Dose of antimicrobial missed/delayed, or their regiment unintentionally ceased
Number of patients

34

10

12

10

\section{Discussion}

The observed reduction in DOT and LOT can be reasonably hypothesized to produce a number of positive downstream effects. There is growing evidence to show that a reduction in antimicrobial use can result in a decrease in AMR, in particular within the hospital setting $[16,17]$. Lower DOT/LOTs reflect reduced unnecessary use of antimicrobials and may lead to reduced antimicrobial resistance rates, reduced Clostridioides difficile infections and reduced infections due to MRSA, carbapenem resistant 
Pseudomonas aeruginosa and extended spectrum beta lactamase (ESBL)-producing enterobacterales [18].

Additionally, AMS programs and interventions that improve antimicrobial prescribing have been shown to increase cure rates, decrease treatment failures and decrease mortality from infection [19]. Of the 34 patients which received broad spectrum antimicrobials which triggered the antibiotic timeout function, 22 (64.7\%) of these either resulted in an intentional discontinuation of therapy or a de-escalation to oral antimicrobials. AMS programs that look to prescribe according to guidelines (including recommended durations) and de-escalate therapy when appropriate have previously demonstrated a $35 \%$ relative risk reduction for mortality associated with guideline-adherent therapy and a $56 \%$ decrease in mortality associated with de-escalation of therapy [20].

The cost saving benefits of AMS intervention tools have been well described with AMS programs reducing antimicrobial costs by an average of $33.9 \%$ and length of stay by $8.9 \%$ [18]. The demonstrated reduction in DOT/LOTs directly reflects reduced antimicrobial use and could be attributed to the antibiotic timeout function prompting treating teams to consider ceasing antimicrobial therapy when indicated. Furthermore, intravenous antimicrobial therapy may often be the rate-limiting step preventing a patient's discharge and the antibiotic timeout function prompting teams to consider an IV-to-oral change in antimicrobial therapy could be a contributing influence [21, 22].

The default number of days an antimicrobial will be prescribed is automatically set to 5 days and unless treating teams are reviewing patient antimicrobials daily, then a patient may have their antimicrobials ceased when there is still an intention to treat. Contributors to this phenomena may include:

time/resource constraints, an accidental oversight when reviewing a patient or teams not knowing that the antibiotic timeout function is utilized in CCLHD hospitals. While cessation of antimicrobials for patients who require them is a serious concern there have been no reported antibiotic timeouts which have resulted in any independently confirmed adverse outcomes for a patient in the CCLHD. Proposed mechanisms to address the potential risks include education during orientation for new clinical teams with intermittent reminders, an automated electronic task generated that prompts teams to review specific antimicrobials about to have the antibiotic timeout function employed, and regular auditing of Incident Information Management System (IIMS) reports regarding antibiotic timeouts.

Another potential negative impact of the hard stop functionality is so called "antibiotic timeout fatigue". The primary purpose of the antibiotic timeout function is to prompt teams to review the appropriateness of antimicrobials periodically. One concern is that if prescribing clinicians are required to repeatedly represcribe antimicrobials they may develop an automated response to resprescribing antimicrobials ceased by the antibiotic timeout function without assessing appropriateness. Alert fatigue is a recurring concern across multiple health professions that utilize patient electronic notes and charts with several studies looking into solutions $[23,24]$. Suggested initiatives include regular education regarding the purpose of the antibiotic timeout function and its demonstrated benefits, adopting a tiered alert system giving greater prominence to antimicrobials involved in more serious infections, junior medical officer 
feedback surveys at the end of each clinical rotation and antimicrobial stewardship teams to monitor for antimicrobials that may have been represcribed without mindful review.

The study has a number of limitations some of which have been outlined above. No direct comparison has been made between the post eMeds cohorts that include and do not include an antibiotic timeout function. As such the effect of the electronic prescribing system on antimicrobial prescribing is difficult to differentiate from the effect of the antibiotic timeout function. It is unclear to what extent changes in general antimicrobial prescribing trends between 2017 and 2019 may have influenced DOT and LOT. Further investigation would be helpful in order to draw further conclusions regarding the demonstrated reduction in DOT and LOT. Patients on concurrent antimicrobials for indications other that CAP or IECOPD treatment were included in the final DOT and LOT count. Whilst this may potentially be considered an effect modifier, antimicrobials were counted in this manner for both pre and post antibiotic timeout cohorts and is unlikely to influence any statistical or practical inferences.

Patients were selected from a pool generated by the restricted antimicrobial software Guidance MS ${ }^{\circledR}$. Antimicrobials are in general more likely to be restricted and require registering on Guidance MS $₫$ if they are broad spectrum and higher risk. These antimicrobials are generally reserved for more severe or unusual infections and thus due to this selection method patients with mild CAP and/or IECOPD were not included. Inclusion of these patients may have revealed further insights into differences/similarities between the two groups. Adjusting the methodology to include patients prescribed non-restricted antimicrobials and those with mild CAP and IECOPD may be an area for future investigation.

A recurring limitation of many antimicrobial stewardship studies is the difficulty in measuring a tangible microbiological benefit. Evidence for the association between the use of antimicrobials and the rise in AMR is well described in laboratory, ecological and human studies at both the individual and population levels [25]. This requires long-term studies with extensive follow up that is outside the scope of this research. Whilst this study did not attempt demonstrate a direct correlation between reduced antimicrobial use and reduced rates of AMR, the existing literature is sufficient to suggest a strong association.

\section{Conclusion}

The electronic prescribing landscape remains ripe with opportunities to integrate AMS tools in an effort to further improve the behaviour of antimicrobial prescribers. The observed reductions in DOT and LOT are clearly suggestive that the antibiotic timeout function has exerted a strong influence on antibiotic prescribing behaviour. It prompts the prescriber to evaluate the patients' current clinical condition and consider consulting therapeutic guidelines in order to examine current practice. The results from this study demonstrate the potential role that antibiotic timeout functionality may have in reducing inappropriate antimicrobial use and improving the culture of antibiotic prescribing in the inpatient environment. There remain a number of challenges including demonstrating broad efficacy of the 
intervention across a range of indications and ensuring that AMS programs aimed at improving antimicrobial prescribing do not adversely affect patient care.

\section{Abbreviations}

AMR

Antimicrobial Resistance

AMS

Antimicrobial Stewardship

ATO

Antibiotic Timeout

CAP

Community Acquired Pneumonia

CCLHD

Central Coast Local Health District

DOT

Days of therapy

eMeds

Electronic Medicine

ESBL

Extended spectrum beta lactamase

ICU

Intensive care Units

IECOPD

Infective Exacerbation of Chronic Obstructive Pulmonary Disease

LOT

Length of therapy

MRSA

Methicillin Resistant Staphylococcus Aureus

NAPS

National Antimicrobial Prescribing Survey

RACF

Residential Aged Care Facility

\section{Declarations}

\section{Ethics approval and consent to participate}

This study had ethics exemption approved by the Central Coast Local Health District Research Governance Office (Reference number: 0121-004C) 


\section{Consent for publication}

Not applicable

\section{Availability of data and material}

Raw data were generated at Central Coast Local Health District. Derived data supporting the findings of this study are available from the corresponding author SG on request.

\section{Competing interests}

Nil

\section{Funding}

Nil

\section{Authors' contributions}

Semun Galimam - data curation (equal), formal analysis (lead), validation (equal), writing - original draft preparation (equal), writing - review \& editing. Brydon Panozzo - data curation (equal), formal analysis (equal), validation (equal), writing - original draft preparation (lead), writing - review \& editing (equal). Kieran Muir - data curation (equal), writing - review \& editing (equal). Ruchir Chavada - conceptualisation (lead), project administration (lead), supervision (lead), validation (lead), writing - original draft preparation (equal).

\section{Acknowledgments}

Nil

\section{References}

1. Laxminarayan R, Van Boeckel T, Frost I, Kariuki S, Khan EA, Limmathurotsakul D, Larsson DJ, LevyHara G, Mendelson M, Outterson K, Peacock SJ. The Lancet Infectious Diseases Commission on antimicrobial resistance: 6 years later. The Lancet Infectious Diseases. 2020 Apr 1;20(4):e51-60.

2. Dyar OJ, Huttner B, Schouten J, Pulcini C. What is antimicrobial stewardship?. Clinical Microbiology and Infection. 2017 Nov 1;23(11):793-8.

3. Baysari MT, Lehnbom EC, Li L, Hargreaves A, Day RO, Westbrook Jl. The effectiveness of information technology to improve antimicrobial prescribing in hospitals: a systematic review and meta-analysis. International journal of medical informatics. 2016 Aug 1;92:15-34.

4. Pearce R, Whyte I. Electronic medication management: is it a silver bullet?. Australian prescriber. 2018 Apr;41(2):32. 
5. Hand KS, Cumming D, Hopkins S, Ewings S, Fox A, Theminimulle S, Porter RJ, Parker N, Munns J, Sheikh A, Keyser T. Electronic prescribing system design priorities for antimicrobial stewardship: a cross-sectional survey of 142 UK infection specialists. Journal of Antimicrobial Chemotherapy. 2017 Apr 1;72(4):1206-16.

6. Timmons V, Townsend J, McKenzie R, Burdalski C, Adams-Sommer V. An evaluation of providerchosen antibiotic indications as a targeted antimicrobial stewardship intervention. American journal of infection control. 2018 Oct 1;46(10):1174-9.

7. Pettit NN, Bhagat P, Nguyen CT, Konold VJ, Kumar M, Choksi A, Pisano J. 1039. Forty-eight-hour Antibiotic Time-out: Impact on Antibiotic Duration and Clinical Outcomes. InOpen Forum Infectious Diseases 2019 Oct 2 (Vol. 6).

8. James C, Coates C, Clay B, Abeles S. Implementation of an Automated Electronic Medical Record "Antibiotic Timeout" Alert at a Tertiary, Academic Medical Center. InOpen Forum Infectious Diseases 2017 (Vol. 4, No. Suppl 1, p. S490). Oxford University Press.

9. Hyak JM, Al Mohajer M, Musher B. 181. Limited Effectiveness of an EMR Alert-Based Antibiotic Timeout Procedure in Solid Tumor Cancer Patients. InOpen Forum Infectious Diseases 2020 Oct (Vol. 7, No. Suppl 1, p. S97). Oxford University Press.

10. Nabovati E, Jeddi FR, Farrahi R, Anvari S. Information technology interventions to improve antibiotic prescribing for patients with acute respiratory infection: a systematic review. Clinical Microbiology and Infection. 2021 Apr 1.

11. Song JH, Thamlikitkul V, Hsueh PR. Clinical and economic burden of community-acquired pneumonia amongst adults in the Asia-Pacific region. International journal of antimicrobial agents. 2011 Aug 1;38(2):108 - 17.

12. Ko FW, Chan KP, Hui DS, Goddard JR, Shaw JG, Reid DW, Yang IA. Acute exacerbation of COPD. Respirology. 2016 Oct;21(7):1152-65.

13. Møller Gundersen K, Nygaard Jensen J, Bjerrum L, Hansen MP. Short-course vs long-course antibiotic treatment for community-acquired pneumonia: A literature review. Basic \& Clinical Pharmacology \& Toxicology. 2019 May;124(5):550-9.

14. Wald-Dickler N, Spellberg B. Short-course antibiotic therapy-replacing constantine units with "shorter is better".

15. Public Health Ontario. Antimicrobial Stewardship Programs (ASPs) Metrics Examples 2017 [Available from: https://www.publichealthontario.ca/-/media/documents/a/2017/asp-metricsexamples.pdf?la=en.

16. Rice LB. Antimicrobial stewardship and antimicrobial resistance. Medical Clinics. 2018 Sep 1;102(5):805 - 18.

17. Beryl P, Baur D, Foschi F, Tacconelli E, editors. Clinical effectiveness of antimicrobial stewardship in reducing antibiotic resistance rate: a metaanalysis. 26th European Congress of Clinical Microbiology and Infectious Diseases 2016 Apr 9-12; Amsterdam, The Netherlands: ESCMID. 
18. Karanika S, Paudel S, Grigoras C, Kalbasi A, Mylonakis E. Systematic review and meta-analysis of clinical and economic outcomes from the implementation of hospital-based antimicrobial stewardship programs. Antimicrobial agents and chemotherapy. 2016 Aug 1;60(8):4840-52.

19. Fishman N. Antimicrobial stewardship. American journal of infection control. 2006 Jun 1;34(5):S5563.

20. Schuts EC, Hulscher ME, Mouton JW, Verduin CM, Stuart JW, Overdiek HW, van der Linden PD, Natsch S, Hertogh CM, Wolfs TF, Schouten JA. Current evidence on hospital antimicrobial stewardship objectives: a systematic review and meta-analysis. The Lancet infectious diseases. $2016 \mathrm{Jul}$ 1;16(7):847 - 56.

21. Davey P, Brown E, Charani E, Fenelon L, Gould IM, Holmes A, Ramsay CR, Wiffen PJ, Wilcox M. Interventions to improve antibiotic prescribing practices for hospital inpatients. Cochrane Database of Systematic Reviews. 2013(4).

22. Engel MF, Postma DF, Hulscher ME, van Berkhout FT, Emmelot-Vonk MH, Sankatsing S, Gaillard CA, Bruns $\mathrm{AH}$, Hoepelman Al, Oosterheert JJ. Barriers to an early switch from intravenous to oral antibiotic therapy in hospitalised patients with CAP. European Respiratory Journal. 2013 Jan 1;41(1):123 - 30 .

23. Carspecken CW, Sharek PJ, Longhurst C, Pageler NM. A clinical case of electronic health record drug alert fatigue: consequences for patient outcome. Pediatrics. 2013 Jun 1;131(6):e1970-3.

24. Kesselheim AS, Cresswell K, Phansalkar S, Bates DW, Sheikh A. Clinical decision support systems could be modified to reduce 'alert fatigue'while still minimizing the risk of litigation. Health affairs. 2011 Dec 1;30(12):2310-7.

25. Tenover FC, McGowan Jr JE. Reasons for the emergence of antibiotic resistance. The American journal of the medical sciences. 1996 Jan 1;311(1):9-16. 